\title{
Highly sensitive, transparent, and flexible gas sensors based on gold nanoparticle decorated carbon nanotubes
}

\author{
Kangho Lee ${ }^{a}$, Vittorio Scardaci ${ }^{a, b}$, Hye-Young Kim ${ }^{\text {a }}$, Toby Hallam ${ }^{a}$, Hugo Nolan ${ }^{\mathrm{a}, \mathrm{c}}$, \\ Brian E. Bolf ${ }^{\mathrm{d}}$, Gregory S. Maltbie ${ }^{\mathrm{d}}$, James E. Abbott ${ }^{\mathrm{d}}$, Georg S. Duesberg ${ }^{\mathrm{a}, \mathrm{c}, *}$ \\ ${ }^{a}$ Centre for Research on Adaptive Nanostructures and Nanodevices (CRANN), Trinity College Dublin, Dublin 2, Ireland \\ ${ }^{b}$ Hewlett-Packard Inkjet Supplies Ireland, Kildare, Ireland \\ 'School of Chemistry, Trinity College Dublin, Dublin 2, Ireland \\ ${ }^{d}$ Hewlett-Packard Printing Technology Development Organization, OR, United States
}

We report on a high performance flexible and transparent chemical sensor based on functionalised single-walled carbon nanotubes (SWCNTs). The SWCNT films were spraydeposited on transparent and flexible plastic substrates, and subsequently decorated with Au nanoparticles (AuNPs) providing a facile and cheap fabrication route. The electrical resistance of the films changed remarkably upon exposure to ammonia (NH3), AuNP decoration enhanced sensitivity to $255 \mathrm{ppb}$ (parts-per-billion), one of the lowest reported so far. The reported sensor performance is a huge improvement towards low power consumption and its room temperature operation augers well for use in mobile devices for environmental protection and air quality control.

Keywords: Chemiresistor; Carbon nanotube; Gold nanoparticle; Polyethylene terephthalate; Ammonia; Sensor

\section{Introduction}

There exists an increasing worldwide demand for sensors, in particular for standalone and mobile systems, which call for small, low power and flexible devices. Generally nitrous oxide $\left(\mathrm{NO}_{x}\right)$ and ammonia $\left(\mathrm{NH}_{3}\right)$ are model compounds for gas sensing due to their oxidising and reducing properties. Metal-oxide-semiconductor (MOS) and solid electrolyte (SE) sensors with typical detection limits of $1-1000 \mathrm{ppm}$ at an operating temperature of a few hundred degrees Celsius are commercially available [1-5]. Although these types of sensors are inexpensive and robust, they do suffer from high energy consumption and cannot be fabricated on flexible substrates.

In recent years, nanowires [6-10] and single-walled carbon nanotubes (SWCNTs) [11-13] have been shown to act as ultrasensitive chemical and biological sensors because of their large surface-to-volume ratio with unique physical and electrical properties. Chemical field-effect transistors (ChemFETs) or chemically modified resistors (chemiresistors), in which nanotubes or nanowires act as the conduction channel between two electrodes,

\footnotetext{
* Corresponding author. Tel.: +353 18963035 .

E-mail address: duesberg@tcd.ie (G.S. Duesberg).
}

work at room temperature and, therefore, have low power consumption. The conductivity of the channel is changed by chemical doping, which can be measured electrically. For SWCNTs, the $\pi$ electron system is fully exposed [14] and, therefore, their electrical properties are extremely sensitive to charge transfer and chemical doping from adsorbing species. So far gas and aqueous solution sensors with detection levels of the order of ppm or sub-ppm have been reported [15]. This matches commercial metal oxide film sensors at room-temperature, avoiding the energy intensive operation at elevated temperatures. Furthermore, SWCNT films can be produced cost effectively by spraying into transparent and flexible films, qualifying them for an extended range of applications.

However, SWCNTs are generally grown as mixtures of semiconducting and metallic nanotubes. This can be critical for sensitivity, reproducibility, and reliability. When using large networks of SWCNTs, this problem averages out to a certain extent. However, for thicker films the detection limit rises as the inner tubes are blocked from interacting with target molecules as they cannot penetrate into the network [11]. Thus, it is important to be able to create thin, but well conducting networks and avoid aggregation of the SWCNTs into bundles during deposition [16]. Despite the low sensitivity of network film structure sensors, their simplicity and low production cost make these devices suitable for fabrication processes. Recently, various functionalisation types such as metal particle $[17,18]$ and 


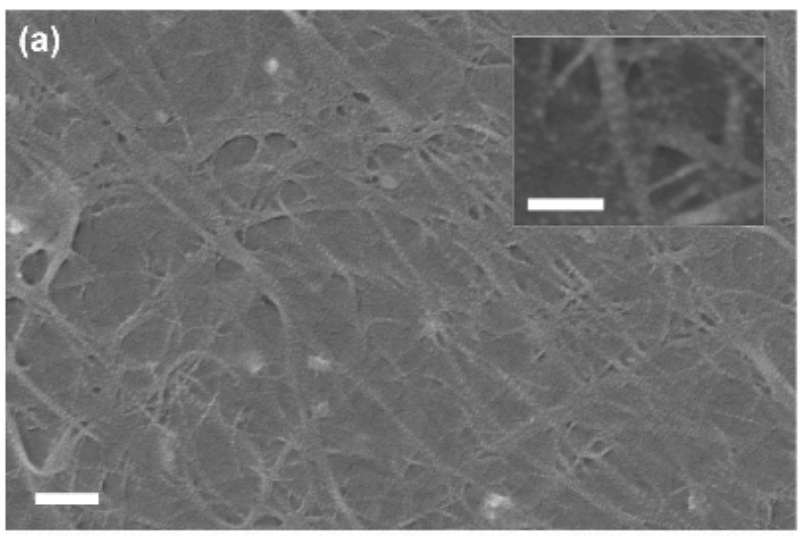

(b)

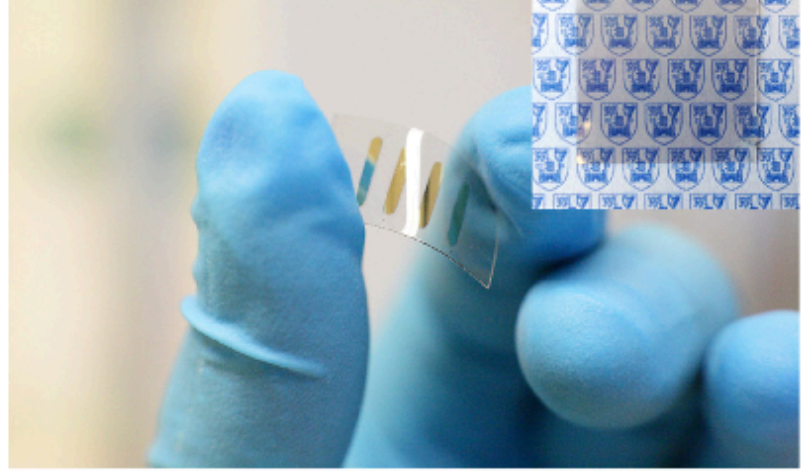

Fig. 1. (a) SEM image of AuNP decorated SWCNT network on the PET substrate. The tiny white dots, indicating Au nanoparticles, typically have a lateral size of $\sim 10 \mathrm{~nm}$, thoroughly covering the surfaces around individual SWCNTs (scale bar $=200 \mathrm{~nm}$ ). Inset: a higher magnification SEM image with arrows indicating AuNP (scale bar $=100 \mathrm{~nm}$ ). (b) Optical images of a transparent and flexible SWCNT network film before (inset) and after electrical contacts deposition. The transferred SWCNT film on the PET substrate is highly flexible and clearly transparent apart from the reflective electrodes.

polymer [19-21] have been reported to improve the performance and sensitivity of nanotube based sensors. It is expected that metal clusters display a full range of reactivity with different molecules and show promise for further functionalisation for high analyte selectivity by attaching specific receptors [22]. Furthermore, transparent and flexible substrates are applicable to SWCNT films for plastic electronics [23].

Here, we present transparent and flexible SWCNT film sensors for the detection of $\mathrm{NH}_{3}$. In the chemiresistor structure, AuNP decorated SWCNT network films are highly sensitive to chemical doping effects. Through the use of polyethylene terephthalate (PET) film support instead of conventional substrates, our sensors are highly flexible and nearly transparent as only the metal electrodes are reflective.

\section{Materials and methods}

Commercial SWCNTs (Iljin Nanotech) were used to make network films. The as-received SWCNTs were dispersed in water using surfactant-assisted ultrasonication, followed by centrifugation to remove agglomerates and, finally, spray-coated onto PET substrates as described in previous work [16]. The as-prepared SWCNT films were decorated by electron-beam evaporation (CHA Mark 50) targeted at $1 \mathrm{~nm}$ Au at a pressure of $\sim 3 \times 10^{-6}$ Torr. As a result, AuNPs with typically lateral size lower than $10 \mathrm{~nm}$ uniformly covered the surfaces around each individual SWCNT (Fig. 1(a)).

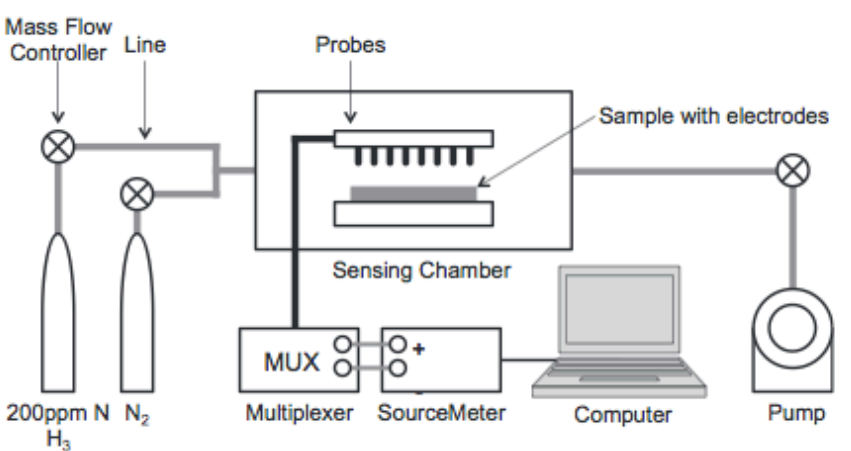

Fig. 2. Schematic of experimental setup for gas sensing.

Electrical contact electrodes of $40 \mathrm{~nm}$ Au atop an adhesion layer of $20 \mathrm{~nm}$ Ni were deposited by sputtering to create intimate electrical contact. For gas sensing tests, the SWCNT film was placed on a custom-made chip-carrier and $\mathrm{Au}$ wires were connected between electrodes of the sensor and bonding pads of the chip-carrier using silver epoxy (DuPont 5007E). As arrayed samples enabled multiple parallel test for improvements of the selectivity and the statistical confidence [24], a multiplexer was attached to our gas sensing system for simultaneous parallel measurement (Fig. 2).

In this study, the AuNP decorated SWCNT films were loaded in a sensing chamber at a pressure of 9.4 Torr and $\mathrm{NH}_{3}$ gas was introduced with dry $\mathrm{N}_{2}$ as carrier gas at a constant flow rate of $100 \mathrm{sccm}$ (standard cubic centimetres per minute). The electrical resistance was measured at a constant bias voltage of $0.1 \mathrm{~V}$ and all sensing tests were carried out at room temperature. In every measurement, the sensor was exposed to dry $\mathrm{N}_{2}$ for $1 \mathrm{~min}$ to record initial resistance and then $\mathrm{NH}_{3}$ balanced with $\mathrm{N}_{2}$ was introduced for $2 \mathrm{~min}$ to observe the sensor response. The sensor was exposed to dry $\mathrm{N}_{2}$ for $5 \mathrm{~min}$ to recover and this gas sensing sequence was periodically repeated four times. Imaging was performed with a Zeiss Ultra HRSEM (high resolution scanning electron microscopy) at an accelerating voltage of $3 \mathrm{keV}$. A thin film of Pt was sputtered onto the samples to avoid charging effects during SEM imaging.

\section{Results and discussion}

Our SWCNT films on PET substrates are transparent and flexible as shown in Fig. 1(b). The photograph shows a bent film on a PET substrate with four Au electrodes clearly visible. The inset shows a film without electrodes on a coloured background underlining its transparency.

The SEM image (Fig. 1(a)) shows a homogenous network of SWCNTs with some small bundles visible. The fine dispersion is due to our optimised spraying process and important for high conductivity and, also, for a high surface exposed for AuNP decoration. The inset shows the film after AuNP decoration. The small white dots are AuNPs with a typical radius of $\sim 5 \mathrm{~nm}$, attached to the circumference of individual SWCNTs or bundles.

The source-drain current versus source-drain voltage $\left(I_{d s}-V_{d s}\right)$ characteristics of a bare SWCNT film and a AuNP decorated SWCNT film are shown in Fig. 3(a). Small differences in the resistance of the SWCNT film in 2-/4-probe configurations indicate that the electrodes are well-defined with low contact resistances between the SWCNT network and metal electrodes as very linear $I_{d s}-V_{d s}$ curves are observed. It is well-known that SWCNTs behave like p-type semiconducting materials in atmosphere, giving good contact to $\mathrm{Ni}$. The resistance of all films is roughly $1.7 \mathrm{k} \Omega$ with an infrared transparency of $>95 \%$ [16]. It is important to note that the high process reliability of our SWCNT films is crucial for 


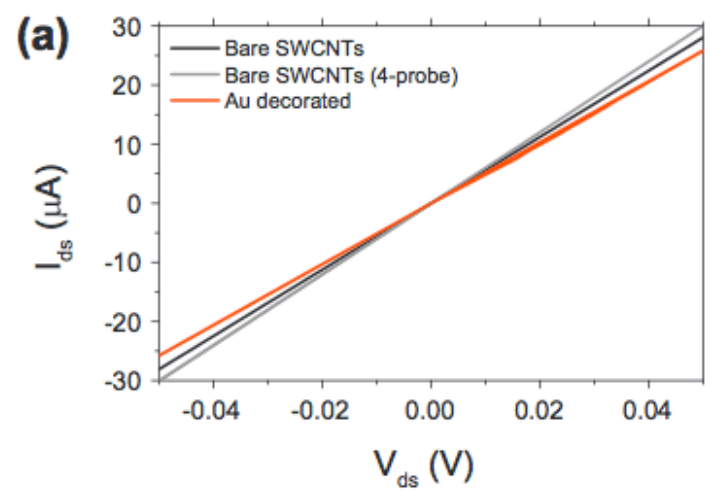

(b)
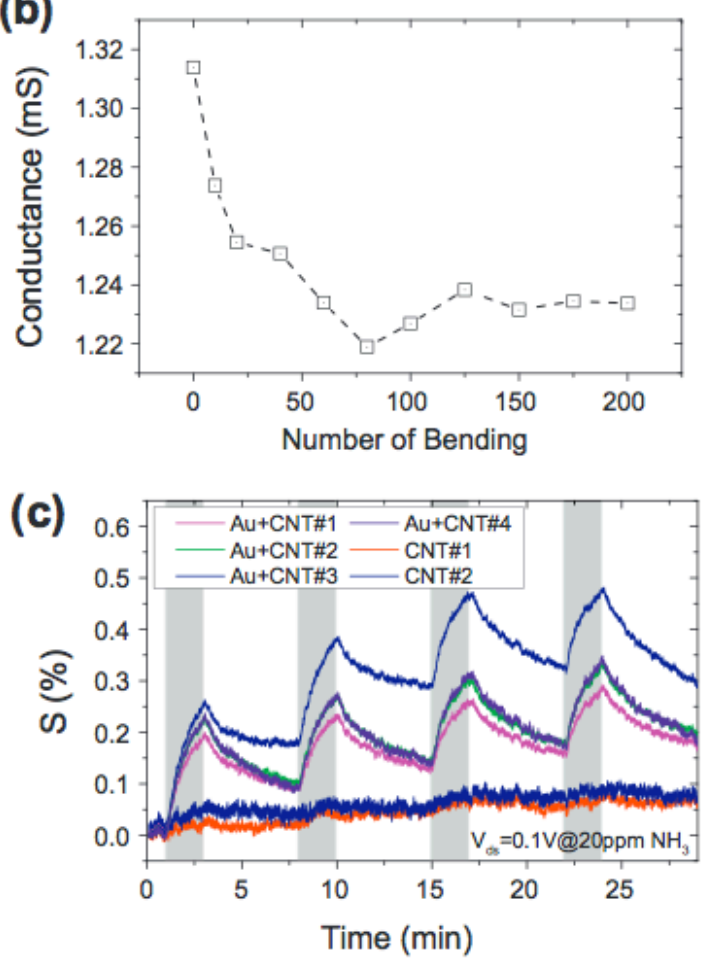

Fig. 3. (a) Source-drain current versus source-drain voltage $\left(I_{\mathrm{ds}}-V_{\mathrm{ds}}\right)$ characteristics of bare SWCNT network film (black and grey lines, respectively) and AuNP decorated SWCNT film (red line). The lower conductance of bare SWCNT film in 4-probe configuration indicates small contact resistance between SWCNT network and electrodes. The resistance of the AuNP decorated SWCNT film is higher than the bare nanotube film. (b) Bending test shows no significant conductance degradation of flexible SWCNT film for two hundred times. (c) Response curves of SWCNT film (red and blue lines) and the AuNP decorated SWCNT film (the other lines) for $20 \mathrm{ppm} \mathrm{NH} \mathrm{N}_{3}$ gas. Light grey boxes stand for periodic introduction of $20 \mathrm{ppm} \mathrm{NH}_{3}$ gas for $2 \mathrm{~min}$. (For interpretation of the references to colour in this figure legend, the reader is referred to the web version of this article.)

their high conductivity and reproducibility. Surprisingly, the AuNP decoration reduced the conductance of the nanotube film. This behaviour has been repeatedly reported and tentatively assigned to AuNPs creating Schottky-type potential barriers at interfaces of the semiconducting nanotubes $[25,26]$. Bending tests were performed to evaluate the stability of conductance of the films. After repeated bending of the flexible substrate two hundred times, the SWCNT film does not show any significant degradation of its conductance (Fig. 3(b)), thus qualifying the films for flexible sensing applications.
The sensing performance is generally evaluated in terms of sensor response and response time. The relative variation of sensor resistance is defined as sensor response $(S)$ given by

$S=\frac{R_{g}-R_{0}}{R_{0}} \times 100 \%$

where $R_{g}$ and $R_{0}$ are the resistances with and without introducing $\mathrm{NH}_{3}$, respectively.

Our sensor test setup enables measurements of multiple samples in parallel. As shown in Fig. 3(c), six responses from different devices on a substrate were recorded at once. Those curves show the sensor responses of the bare (lower two curves) and AuNP decorated SWCNT films (the other curves) upon $20 \mathrm{ppm}$ of $\mathrm{NH}_{3}$ exposure for four cycles of gas injection and recovery.

The two bare films and the four decorated films show very similar responses to the $\mathrm{NH}_{3}$ injections, underlining the high reproducibility of our films. Only one of the four AuNP decorated films (denoted as $\mathrm{Au}+\mathrm{CNT \# 3}$ ) showed a less complete recovery after the first $\mathrm{NH}_{3}$ injection. The AuNP decorated films show a much stronger sensitivity to the gas exposure, as the higher amplitude of $S$ indicates. This may be explained as the following.

When the bare SWCNT films are exposed to $\mathrm{NH}_{3}$, it adsorbs on the surface of SWCNTs and acts as an electron-donor. It has been estimated that each $\mathrm{NH}_{3}$ molecule donates 0.03-0.04 electrons with binding energy of $0.15-0.18 \mathrm{eV}$, to the nanotube $[27,28]$. $\mathrm{NH}_{3}$ molecules effectively shift the transfer characteristics of SWCNTs to negative, thus the conductance of the SWCNTs decreases as the valance band is shifted away from the Fermi level which results in hole depletion. The sensor response to $\mathrm{NH}_{3}$ has been controversial as no or low binding energy is estimated [29]. Through interaction with the hydroxyl groups on the $\mathrm{SiO}_{2}$ substrate, the electronic structure of SWCNTs is considered to be indirectly influenced by $\mathrm{NH}_{3}$. However, by using a PET substrate, we eliminate this contribution from $\mathrm{SiO}_{2}$ substrates. Therefore, we investigated the substantial effects on the nanotube film from $\mathrm{NH}_{3}$. Decorated metal nanoparticles form a Schottky-type potential barrier $\phi_{B}$ on semiconducting materials $[25,26]$. This barrier is sensitive to the metal work function which is well-known to be modified by adsorbed gaseous molecules. Though $\mathrm{NH}_{3}$ molecules adsorbed onto the nanotubes are also able to modify the work function of SWCNTs, they cannot affect this interfacial potential barrier [30].

Fig. 4(a) and (b) show typical gas response curves obtained by exposing AuNP decorated SWCNT film sensors to various concentrations of $\mathrm{NH}_{3}$. Even at sub-ppm level, peaks were clearly distinguishable. The sensor slowly recovers in pure $\mathrm{N}_{2}$ at room temperature, thus the curves show a drift. This could be avoided by UV light illumination or annealing [31-33]. The gas response curves drastically increase $\sim 20 \mathrm{~s}$ after the initial gas introduction, which is attributed to the relatively high volume of the gas sensing setup.

The sensitivity of our sensors is defined as percentile resistance change between initial sensor resistance and the maximum value at the peak of the first gas sensing cycle. The AuNP decorated SWCNT films show much higher sensitivity than bare films. Metal nanoparticle decoration strongly affects the charge transfer characteristics of SWCNT as it creates localised depletion regions that act as charge scattering sites and change the potential barrier at the SWCNT-nanoparticle interface $[17,18,26]$. The lower conductivity of the SWCNT film upon AuNP decoration can be understood by the introduction of depletion zones through the AuNPs into the one dimensional channel of the SWCNTs $[26,34]$. Electron donation of adsorbed $\mathrm{NH}_{3}$ molecules on the AuNPs adds electronic density and hole-electron recombination subsequently occurs after crossing over the interfacial potential barrier between the AuNP and the SWCNT, leading to a reduction of the hole current through 

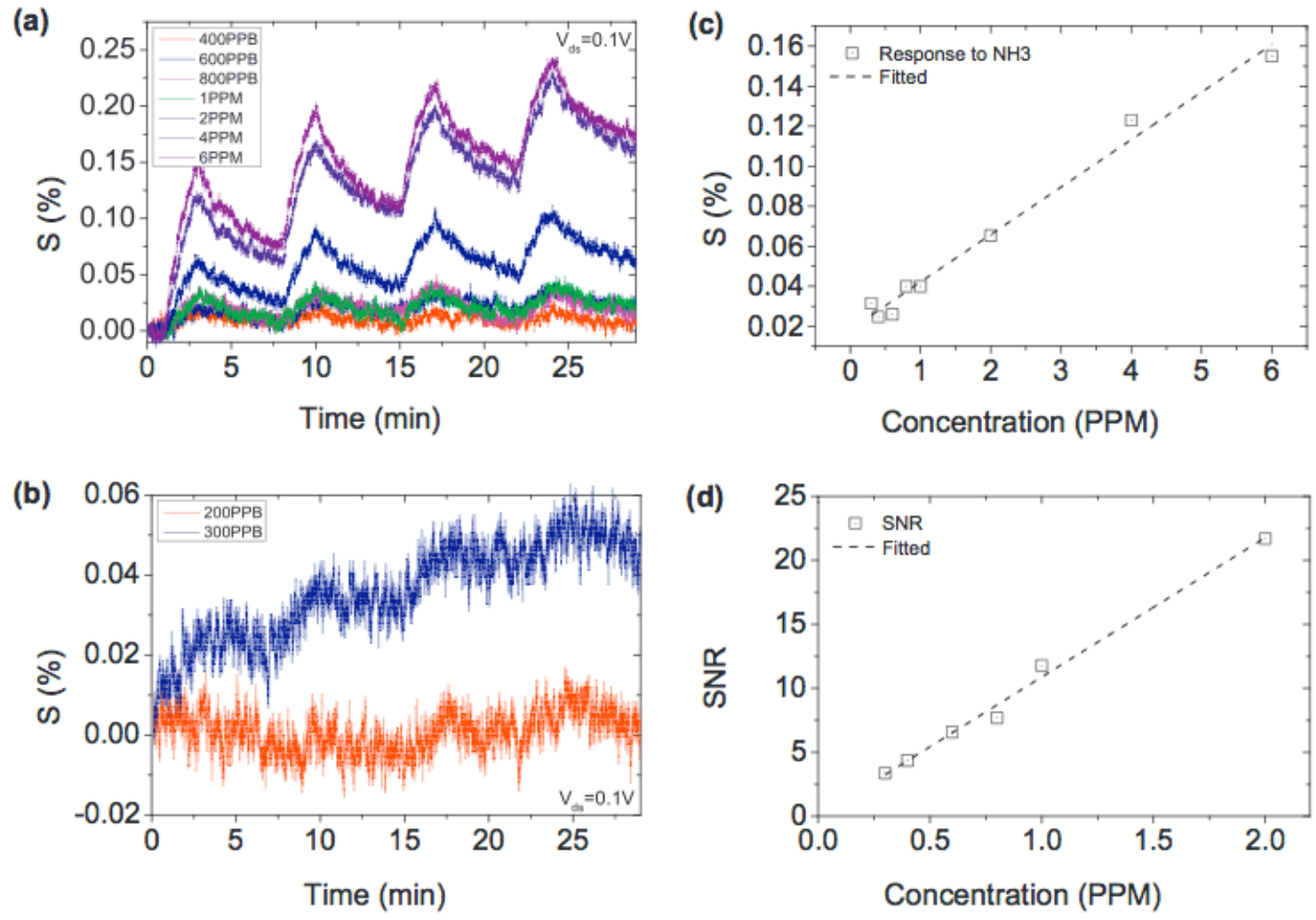

Fig. 4. Sensor response curves of AuNP decorated SWCNT films at different $\mathrm{NH}_{3}$ concentration (a) from $400 \mathrm{ppb}$ to $6 \mathrm{ppm}$ and of (b) $300 \mathrm{ppb}$. Light grey boxes stand for periodic introduction of $\mathrm{NH}_{3}$ gas for $2 \mathrm{~min}$. (c) Plots of sensitivity indicating percentile resistance changes between initial resistance and peak value of the first gas sensing cycle. (d) Signal-to-noise ratio (SNR) of sensor is proportionally corresponding to $\mathrm{NH}_{3}$ concentration below $2 \mathrm{ppm}$.

the SWCNT. As a result, electron-hole recombination at the potential barrier gives rise to a negative gate voltage shift resulting in improved sensor response. Whilst Schottky barriers are known to depend on work function and gas exposure, fundamental electronic interactions between SWCNTs and metal nanoparticles and the description of the potential barrier at nanoparticle interfaces are still incomplete.

The sensitivities at different gas concentrations are shown in Fig. 4(c). This time differential sensitivities are linearly proportional to the gas concentration, which allows determination of the $\mathrm{NH}_{3}$ gas concentration easily. The theoretical limit of detection (LOD) of sensors can be estimated by the relative resistance change in the baseline using the root-mean-square (rms) derivation. We took about 800 data points within the first minute before gas introduction to get the initial resistance of AuNP decorated SWCNT film. The rms noise of the sensor was calculated using the following equation,

$\mathrm{rms}_{\text {noise }}=\sqrt{\frac{\sum\left(R_{\text {sensor }}-R_{0}\right)^{2}}{N}}$

where $R_{\text {sensor }}, R_{0}$, and $N$ are measured resistance depending on time, the initial resistance of the sensor, and the number of data points, respectively. As depicted in Fig. 4(d), signal-to-noise ratio (SNR) is linearly proportional to $\mathrm{NH}_{3}$ gas concentration below $2 \mathrm{ppm}$. The amplitude of signal should be at least three times larger than the noise to be distinguishable [35]. As a result, the LOD of the AuNP decorated SWCNT sensors is $255 \pm 20 \mathrm{ppb}$ in our measurement setup. In fact, it was difficult to recognise peaks at $200 \mathrm{ppb}$ whilst they were obvious above $300 \mathrm{ppb}$ as seen in Fig. 4(b). In other words, the theoretical estimation of the sensor successfully corresponds with the experimental results.

\section{Conclusion}

We demonstrated that transparent, flexible, and highly conductive SWCNT films on PET can be fabricated in a reproducible manner. We investigated their use as gas sensors and showed that they can act as low power $\mathrm{NH}_{3}$ detectors. We demonstrated that the AuNP decoration on the transparent SWCNT films by electronbeam evaporation improved the gas sensor performance. Multiple devices measured in parallel showed similar responses. This is tentatively assigned to the modification of carrier depletion zones induced by the deposited AuNPs on the SWCNTs. They showed a fast response time to $\mathrm{NH}_{3}$; but, however, did not fully recover at room temperature. A detection level on the order of $255 \mathrm{ppb}$ at room temperature was derived, and a corresponding value was experimentally achieved, which to the best of our knowledge, is one of the lowest values reported for nanotube derived sensors.

\section{Acknowledgements}

This research was supported by the Science Foundation Ireland (SFI) under contract no. 08/CE/I1432_S1, between Trinity College CRANN and Hewlett Packard and also for CSET 08/CE/I1432. G.S.D. acknowledges SFI for PICA PI_10/IN.1/13030. All microscopy images were taken by the Advanced Microscopy Laboratory (AML) facility, CRANN, Trinity College Dublin.

\section{References}

[1] M.J. Madou, S.R. Morrison, Chemical Sensing with Solid State Devices, Academic Press, New York, 1989

[2] T. Seiyama (Ed.), Chemical Sensor Technology, 1, Elsevier, New York, 1988. 
[3] G. Korotcenkoy, Metal oxides for solid-state gas sensors: what determines our choice? Materials Science and Engineering: B 139 (2007) 1-23.

[4] J.W. Fergus, Solid electrolyte based sensors for the measurement of CO and hydrocarbon gases, Sensors and Actuators B: Chemical 122 (2007) 683-693.

[5] B.Timmer, W. Olthuis, A. Van Den Berg, Ammonia sensors and their applications - a review, Sensors and Actuators B: Chemical 107 (2005) 666-677.

[6] Y. Cui, Q. Wei, H. Park, C.M. Lieber, Nanowire nanosensors for highly sensitive and selective detection of biological and chemical species, Science 293 (2001) 1289-1292.

[7] F. Favier, E.C. Walter, M.P. Zach, T. Benter, R.M. Penner, Hydrogen sensors and switches from electrodeposited palladium mesowire arrays, Science 293(2001) 2227-2231.

[8] a. Kolmakov, Y. Zhang, G. Cheng, M. Moskovits, Detection of $\mathrm{CO}$ and $\mathrm{O}_{2}$ using tin oxide nanowire sensors, Advanced Materials 15 (2003) 997-1000.

[9] C. Li, D. Zhang, X. Liu, S. Han, T. Tang, J. Han, et al., $\mathrm{In}_{2} \mathrm{O}_{3}$ nanowires as chemical sensors, Applied Physics Letters 82 (2003) 1613-1615.

[10] Q. Wan, Q.H. Li, Y.J. Chen, T.H. Wang, X.L. He, J.P. Li, et al., Fabrication and ethano sensing characteristics of $\mathrm{ZnO}$ nanowire gas sensors, Applied Physics Letters 84 (2004) 3654-3656.

[11] J. Kong, N.R. Franklin, C. Zhou, M.G. Chapline, S. Peng, K. Cho, et al., Nanotube molecular wires as chemical sensors, Science 287 (2000) 622-625.

[12] A. Star, J.P. Gabriel, K. Bradley, G. Grüner, Electronic detection of specific protein binding using nanotube FET devices, Nano Letters 3 (2003) 459-463.

[13] G. a Zelada-Guillén, J. Riu, A. Düzgün, F.X. Rius, Immediate detection of living bacteria at ultralow concentrations using a carbon nanotube based potentiometric aptasensor, Angewandte Chemie 48 (2009) 7334-7337.

[14] M. Meyyappan, Carbon Nanotubes: Science and Applications, CRC, Press, Boca Raton, FL, 2005.

[15] T. Zhang, S. Mubeen, N.V. Myung, M. a Deshusses, Recent progress in carbon nanotube-based gas sensors, Nanotechnology 19 (2008) 332001.

[16] V. Scardaci, R. Coull, J.N. Coleman, Very thin transparent, conductive carbon nanotube films on flexible substrates, Applied Physics Letters 97 (2010) 023114.

[17] Y. Sun, H.H. Wang, High-performance flexible hydrogen sensors that use carbon nanotubes decorated with palladium nanoparticles, Advanced Materials 19 (2007) 2818-2823.

[18] S. Mubeen, T. Zhang, N. Chartuprayoon, Y. Rheem, A. Mulchandani, N.V. Myung, et al., Sensitive detection of $\mathrm{H}_{2} \mathrm{~S}$ using gold nanoparticle decorated singlewalled carbon nanotubes, Analytical Chemistry 82 (2010) 250-257.

[19] P. Qi, O. Vermesh, M. Grecu, A. Javey, Q. Wang, H. Dai, et al., Toward large arrays of multiplex functionalized carbon nanotube sensors for highly sensitive and selective molecular detection, Nano Letters 3 (2003) 347-351.

[20] T. Zhang, M.B. Nix, B.-Y. Yoo, M.A. Deshusses, N.V. Myung, Electrochemically functionalized single-walled carbon nanotube gas sensor, Electroanalysis 18 (2006) 1153-1158.

[21] T. Zhang, S. Mubeen, E. Bekyarova, B.Y. Yoo, R.C. Haddon, N.V. Myung, et al., Poly(m-aminobenzene sulfonic acid) functionalized single-walled carbon nanotubes based gas sensor, Nanotechnology 18(2007) 165504.

[22] Q. Zhao, M. Buongiorno Nardelli, W. Lu, J. Bernholc, Carbon nanotube-metal cluster composites: a new road to chemical sensors? Nano Letters 5 (2005) 847-851.

[23] E. Artukovic, M. Kaempgen, D.S. Hecht, S. Roth, G. Grüner, Transparent and flexible carbon nanotube transistors, Nano Letters 5 (2005) 757-760.

[24] R. Daly, S. Kumar, G. Lukacs, K. Lee, A. Weidlich, M. Hegner, et al., Cell proliferation tracking using graphene sensor arrays, Journal of Sensors 2012 (2012) 219485.

[25] F. Ruffino, M.G. Grimaldi, F. Giannazzo, F. Roccaforte, V. Raineri, Size-dependent Schottky barrier height in self-assembled gold nanoparticles, Applied Physics Letters 89 (2006) 243113

[26] D.R. Kauffman, A. Star, Chemically induced potential barriers at the carbon nanotube-metal nanoparticle interface, Nano Letters 7 (2007) 1863-1868.

[27] H. Chang, J. Do Lee, S.M. Lee, Y.H. Lee, Adsorption of $\mathrm{NH}_{3}$ and $\mathrm{NO}_{2}$ molecules on carbon nanotubes, Applied Physics Letters 79 (2001) 3863-3865.

[28] J. Zhao, A. Buldum, J. Han, J.P. Lu, Gas molecule adsorption in carbon nanotubes and nanotube bundles, Nanotechnology 13 (2002) 195-200.

[29] D.R. Kauffman, A. Star, Carbon nanotube gas and vapor sensors, Angewandte Chemie 47 (2008) 6550-6570

[30] J. Suehiro, H. Imakiire, S. Hidaka, W. Ding, G. Zhou, K. Imasaka, et al., Schottkytype response of carbon nanotube $\mathrm{NO}_{2}$ gas sensor fabricated onto aluminum electrodes by dielectrophoresis, Sensors and Actuators B: Chemical 114 (2006) 943-949.

[31] J. Li, Y. Lu, Q. Ye, M. Cinke, J. Han, M. Meyyappan, Carbon nanotube sensors for gas and organic vapor detection, Nano Letters 3 (2003) 929-933.
[32] F. Schedin, K. a, S, Geim, V. Morozov, E.W. Hill, P. Blake, et al., Detection of individual gas molecules adsorbed on graphene, Nature Materials 6 (2007) 652-655

[33] G. Ko, H.-Y. Kim, J. Ahn, Y.-M. Park, K.-Y. Lee, J. Kim, Graphene-based nitrogen dioxide gas sensors, Current Applied Physics 10 (2010) 1002-1004.

[34] J.-H. Yun, J. Kim, Y.C. Park, J.-W. Song, D.-H. Shin, C.-S. Han, Highly sensitive carbon nanotube-embedding gas sensors operating at atmospheric pressure, Nanotechnology 20 (2009) 055503.

[35] L.A. Currie, Nomenclature in evaluation of analytical methods including detection and quantification capabilities, Pure and Applied Chemistry 67 (1995) 1699-1723.

\section{Biographies}

Kangho Lee obtained his BS, MS, and PhD in Electrical Engineering from Korea University with Prof. Gyu-Tae Kim in 2005, 2007, and 2012. He is currently studying for postdoctoral research at Centre for Research on Adaptive Nanostructures and Nanodevices (CRANN), Trinity College Dublin. His research interest is low dimensional nanomaterials with structuring and analysis.

Vittorio Scardaci obtained his BSc and MSc in Industrial Chemistry from Catania University, Italy in 2004 and his PhD from Cambridge University (UK) in 2009. He currently works as R\&D scientist at Hewlett-Packard Ireland.

Hye-Young Kim obtained her BS, MS, and PhD in Electrical Engineering from Korea University with Prof. Gyu-Tae Kim in 2004, 2007, and 2011. She is currently studying for postdoctoral research at CRANN, Trinity College Dublin. Her research interest is fabrication and analysis of nanodevices.

Toby Hallam received his BSc and PhD at the University of New South Wales (UNSW) while working in the Centre for Quantum Computer Technology (COCT) focusing on a bottom up approach to single dopant placement for a solid-state quantum computer. He subsequently worked at the Cavendish Laboratories investigating state of the art polymer semiconductors using electrical scanning probe techniques. He has since worked at CRANN, Trinity College Dublin. There his work has focused on Soft Lithography processing of graphene and other 2D materials.

Hugo Nolan received his BA (mod) in Physics and Chemistry of Advanced Materials from Trinity College Dublin in 2010. He is currently working towards his $\mathrm{PhD}$ in the CRANN in Trinity College Dublin. His research primarily focusses on the synthesis of graphene and related carbon nanomaterials through CVD techniques and their functionalisation and modification using plasma methods towards implementation in device applications.

Brian E. Bolf has been working for HP for 17 years and as an R \& D technician for the last 15 years. His current focus is on thin film material deposition using sputtering and evaporation techniques. He is the lead technician for evaporative thin film deposition at the HP printing site in Corvallis, Oregon.

Gregory S. Maltbie has been working at HP as a thin films technician for 19 years. He has been working in R\&D thin film deposition for the last four years; including deposition work using thermal and e-beam evaporation.

James E. Abbott obtained his BS degrees in Physics and Engineering Physics in 1997 and MS and PhD in Physical Chemistry in 2002 and 2007 from Oregon State University. He was an instructor and director of enlisted physics instruction for the United States Navy 2002-2006. He is currently the technical lead for thin film materials development in the HP printing division based in Corvallis, Oregon. His technical interests are focused on the development of thin film materials and processes.

Georg S. Duesberg graduated in Physical Chemistry from the University of Kassel, Germany in 1996. He moved on to the Max-Planck-Institute for Solid State Research, Stuttgart and Trinity College Dublin to receive his PhD in 2001. From 2001 to 2005, he was in the Corporate Research Department at the Infineon AG, Munich, Germany. From 2005 to 2007, Prof. Duesberg worked in the Thin Films Department, Future Furnace, of the Qimonda AG, Dresden, Germany. In July 2007, he took on a position as a Principal Investigator in CRANN in Trinity College Dublin, Ireland. Since July 2011, he is Professor in the School of Chemistry and is currently Director of Research. Prof. Duesberg has co-authored more than 120 publications with more than 5000 citations and holds more than 25 patents. 